\title{
Long-term expression of zinc transporters in hippocampus following penicillin-induced developmental seizures and its regulation by E-64d
}

\author{
HONG NI $^{1}$, LE-LING ZHANG ${ }^{1}$, SHOU-YUN REN ${ }^{1}$ and BAO-LIANG SUN ${ }^{2}$ \\ ${ }^{1}$ Neurology Laboratory, Institute of Pediatrics, Children's Hospital of Soochow University, Suzhou, Jiangsu 215003; \\ ${ }^{2}$ Key Laboratory of Cerebral Microcirculation in Universities of Shandong, Department of Neurology, \\ Affiliated Hospital of Taishan Medical College, Taian, Shandong 271000, P.R. China
}

Received January 14, 2015; Accepted February 19, 2016

DOI: $10.3892 /$ etm.2016.3276

\begin{abstract}
Autophagy has been shown to be involved in the pathophysiology of developmental seizure-induced brain damage. The present study aimed to examine whether E-64d, an autophagy inhibitor, was able to facilitate developmental seizure-induced hippocampal mossy fiber sprouting, in particular sprouting-associated zinc transporter signals. Recurrent seizures were induced by penicillin every other day in Sprague-Dawley rats from postnatal day 21 (P21). Rats were randomly assigned into the control group (CONT), recurrent seizure group (RS) and the seizure plus E-64d group (E64D). The expression levels of beclin-1 and B-cell lymphoma 2 were analyzed at 1.5, 3, 6 and $24 \mathrm{~h}$ after the last seizures using western blot analysis. At P51, mossy fiber sprouting and the mRNA expression levels of zinc transporter 2 (ZnT-2), ZnT-4, ZnT-5, ZnT-6, ZnT-7, divalent cation transporter 1, Zrt-Irt-like protein 6 (ZIP-6), ZIP-7, cathepsin D and cathepsin L in the rat hippocampus were assessed using Timm staining and reverse transcription-quantitative polymerase chain reaction analysis, respectively. Reduced hippocampal mossy fiber sprouting were detected in the E-64d-treated rats compared with the non-treated control. In parallel with these observations, there was a marked reduction in the mRNA expression levels of ZnT-4 at P51 in the E-64d-treated rat hippocampus compared with the non-treated seizure group. Linear correlation analysis showed significant inter-relationship among ZIP-7, ZnT-4, ZnT-5, ZnT-7, cathepsin D and cathepsin L. These results indicate that the ZnT-4/ZIP-7/cathepsin signaling pathway serves a crucial function in the neuroprotective effects of E-64d. Thus, E-64d may offer a novel strategy for the development of therapeutic interventions for developmental seizure-induced brain damage.
\end{abstract}

Correspondence to: $\mathrm{Dr}$ Hong Ni, Neurology Laboratory, Institute of Pediatrics, Children's Hospital of Soochow University, 303 Jingde Road, Suzhou, Jiangsu 215003, P.R. China

E-mail: nyr2000@yeah.net

Key words: zinc transporter, mossy fiber spouting, E-64d, developmental, seizure

\section{Introduction}

Early intervention in developmental seizure-induced brain damage is a focus area in recent pediatric clinical and animal studies. The autophagic/lysosomal pathway has been recently considered as a promising therapy for preventing and treating neurodegenerative diseases, in addition to seizure-induced brain injuries (1-3). However, the underlying molecular mechanism of its efficacy remain unclear.

Developmental seizure-induced long-term adverse effects include neurodysfunction and pathological phenomena such as regenerative aberrant mossy fiber sprouting in the hippocampus, which is positively correlated with long-term neurofunctional impairment (4-6). Hippocampal mossy fibers are the axons of dentate gyrus granule cells. Overstimulation of mossy fibers in epileptic activities leads to aberrant regenerative sprouting, which is associated with aberrant zinc homeostasis $(7,8)$. In a previous study the present authors showed that recurrent neonatal seizures could cause long-term cognitive dysfunction and disturbed hippocampal expression of plasticity related gene 1 (PRG-1), which could be reversed by pretreatment with the autophagy inhibitor cathepsin B inhibitor (CBI) (9). The results of another study showed that the autophagy inhibitor 3-methyladenine (3-MA) regulated the acute phase expression of autophagy and zinc transporter-associated genes at 1.5, 3, 6 and $24 \mathrm{~h}$ after the last seizures (10). These results showed linear correlations among microtubule-associated protein light chain 3 (LC3), beclin-1, zinc transporter 1 (ZnT-1) and ZnT-2 transcripts in the hippocampus and cerebral cortex in the recurrent seizure group, which could be modulated by 3-MA. However, there are no reports investigating the long-term alterations in the mRNA expression levels of $\mathrm{ZnT}$ signals in hippocampus pretreatment with autophagy inhibitor following developmental seizures.

The aim of the present study was to characterize the long-term expression profiles of ZnT-associated genes in the hippocampus following developmental seizures. It was hypothesized that the disturbed expression of ZnTs would correlate with increases in hippocampal axon sprouting which could be inhibited by pretreatment with E-64d, another autophagy inhibitor. 


\section{Materials and methods}

Animals and grouping. A total of 130 Sprague-Dawley male rats (obtained from the Experimental Animal Center of Zhejiang University, Hangzhou, China) at postnatal day 21 (P21) were used in all experiments. Animals were treated in accordance with the guidelines set by the National Institutes of Health for the humane treatment of animals and were approved by the animal care and use committee of Soochow University. Attempts were made to minimize the number of animals used. Rats were randomly assigned to three groups: Control (CONT; n=30); penicillin-induced recurrent seizures group ( $\mathrm{RS} ; \mathrm{n}=50)$; and the E-64d-treated seizure group (E64D; n=50). Penicillin (5.1 U/mg dissolved in saline; RS and E64D; North China Pharmaceutical Co., Ltd., Shijiazhuang, China) or saline (CONT) was administered intraperitoneally (i.p.) to induce seizure, as previously reported (8). Penicillin was injected every other day for 11 consecutive days from postnatal day 21 (P21). E64D group rats were pretreated with E-64d (Sigma-Aldrich, St. Louis, MO, USA) prior to seizure induction ( $4 \mu 1,1 \mathrm{mg} / \mu \mathrm{l} / \mathrm{day}$, i.p.). Thirty-four animals died due to serious generalized seizures. The animals suffering generalized seizures (stages 5 and 6 , bilateral forelimb clonus with rearing and loss of postural control) (8) were used for subsequent experiments ( $\mathrm{n}=30$ per seizure group).

Western blot analysis. At 3, 6 and $24 \mathrm{~h}$ after the final seizure, rats ( $n=30$ per group) were sacrificed by $4 \%$ chloral hydrate (1 ml/100 g i.p.; Sinopharm Chemical Reagent Co., Ltd., Shanghai, China; cat. no. 30037517) and the hippocampus tissues in every time point from each group $(n=6)$ were rapidly removed and immediately frozen in dry ice for western blot analysis, as described previously by Luo et al (11). Briefly, samples were homogenized in western blot analysis buffer containing $10 \mathrm{mM}$ Tris- $\mathrm{HCl}$ (pH 7.4), $150 \mathrm{mM} \mathrm{NaCl}, 1 \% \mathrm{v} / \mathrm{v}$ Triton X-100, 1\% sodium deoxycholate, 5 mM EDTA (all chemicals obtained from Sigma-Aldrich). The homogenate was then centrifuged at $10,000 \mathrm{rpm}$ for $10 \mathrm{~min}$ at $4^{\circ} \mathrm{C}$ to obtain the supernatant, which was stored at $-70^{\circ} \mathrm{C}$ until further use. Subsequently, $30 \mu \mathrm{g}$ protein from each sample was subjected to $10 \%$ SDS-PAGE. After blocking overnight at $4^{\circ} \mathrm{C}$ with skimmed milk (cat. no. 232100; BD Biosciences, Franklin Lakes, NJ, USA), the blots were incubated with one of the following antibodies: Goat polyclonal anti-beclin-1 (1:1,000; cat. no. sc-11427; Santa Cruz Biotechnology, Inc., Dallas, TX, USA) and rabbit polyclonal anti-B-cell lymphoma 2 (Bcl-2; 1:100; cat. no. sc-492; Santa Cruz Biotechnology, Inc.) in Tris-buffered saline containing $0.2 \%$ Tween-20 (TBST; Sinopharm Chemical Reagent Co., Ltd.; cat. no. 30189328) and $5 \%$ nonfat dry milk overnight at $4^{\circ} \mathrm{C}$. Following overnight incubation, membranes were washed and incubated with horseradish peroxidase-conjugated secondary antibody in TBST for $2 \mathrm{~h}$ (goat anti-rabbit IgG and rabbit anti-goat IgG; cat. no. GAR0072 and RAG0072, respectively; dilution, 1:3,000; MultiSciences Biotech Co., Ltd., Hangzhou, China). Immunoreactivity was detected using enhanced chemiluminescent autoradiography (Western BrightECL kit; cat. no. k-12045-D50; Advansta Inc., Menlo Park, CA, USA). The relative changes in the intensity of each immunoreactive band were evaluated using SigmaScan Pro 5.0 (Systat
Software, Inc., San Jose, CA, UK) and were normalized against the loading control $\beta$-actin.

Timm staining. A subset of rats ( $\mathrm{n}=6$ each group) underwent Timm staining on P51 according to the method previously described (12). The contents of the Timm staining solution were obtained from Sinopharm Chemical Reagent Co., Ltd., and were as follows: Gum arabic powder (cat. no. 9000-01-5); hydroquinone (cat. no. 10011317); citric acid monohydrate (cat. no. 10007118); trisodium citrate dihydrate (cat. no. 10019418); and silver nitrate (cat. no. 10018461). Sprouting was analyzed at a magnification of x10 using an Olympus CX-40 light microscope (Olympus Corp., Tokyo, Japan).

Reverse transcription-quantitative polymerase chain reaction $(R T-q P C R)$. A total of 6 rats from each group were sacrificed using $4 \%$ chloral hydrate ( $1 \mathrm{ml} / 100 \mathrm{~g}$ i.p.) at P51. Hippocampal samples from each group were subjected to RT-qPCR analysis, as described previously (8). Briefly, total RNA was extracted using TRIzol reagent (Invitrogen; Thermo Fisher Scientific, Inc., Waltham, MA, USA) according to the manufacturer's instructions. The concentration, purity and quantity of the total isolated RNA was determined by measuring the optical density at 260 and $280 \mathrm{~nm}$ by UV spectrophotometry using a NanoPhotometer UV/Vis spectrophotometer (Implen, Munich, Germany). Next, $2 \mu \mathrm{g}$ total RNA was reverse transcribed into cDNA using $1 \mu \mathrm{g}$ random primers (Promega Corp., Madison, WI, USA), $1.5 \mu 1$ M-MLV Reverse Transcriptase and $0.6 \mu 1$ RNase inhibitor (Thermo Fisher Scientific, Inc.). For RT, the total reaction volume was $40 \mu \mathrm{l}$ and the sample was incubated at $37^{\circ}$ for $60 \mathrm{~min}$, followed by $95^{\circ} \mathrm{C}$ for $5 \mathrm{~min}$. Subsequently, qPCR was performed with an Applied Biosystems 7500 Real-Time PCR system (Thermo Fisher Scientific, Inc.) using TaqMan Gene Expression Master Mix (Thermo Fisher Scientific, Inc.). The total volume of the PCR reaction mixture

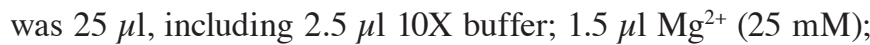
$0.5 \mu \mathrm{l}$ dNTP (10 mM); $0.25 \mu \mathrm{l}$ hot start Taq DNA polymerase; $0.5 \mu 1$ of each primer; and $0.3 \mu 1$ TaqMan probe. The primers and probes used in qPCR for the zinc transporter genes (ZnT-2, ZnT-4, ZnT-5, ZnT-6 and ZnT-7), divalent cation transporter 1 (DCT-1), Zrt-Irt-like protein-6 (ZIP-6), ZIP-7, cathepsin D and cathepsin $\mathrm{L}$ were designed against the GenBank sequences using the Primer Express 2.0 software (Applied Biosystems; Thermo Fisher Scientific, Inc.), and are shown in Table I. The PCR cycling conditions were $95^{\circ} \mathrm{C}$ for $10 \mathrm{~min}$ (initial denaturation), and 40 cycles of $95^{\circ} \mathrm{C}$ for $15 \mathrm{sec}$ and $60^{\circ} \mathrm{C}$ for $60 \mathrm{sec}$. Quantitative fluorescent data were collected and analyzed using SDS software, version 1.3 (Thermo Fisher Scientific, Inc.). Threshold cycle data were determined by setting the default threshold to 0.08; when the fluorescence intensity reaches this threshold value, the PCR cycle number was regarded to be the threshold cycle $(\mathrm{Cq})$ value. Initially, the RT-qPCR Cq value of the target mRNAs and the internal control ( $\beta$-actin) were determined, and then the $\Delta \mathrm{Cq}$ method of relative quantification was used to determine the fold changes in expression. The fold change in expression was obtained using the $2^{-\Delta \Delta \mathrm{Cq}}$ method (13).

Statistical analysis. The protein and mRNA expression levels $\left(2^{-\Delta \Delta C q}\right)$ were analyzed with post-hoc comparisons using analysis of variance, followed by Bonferroni test. Timm staining 
Table I. Oligonucleotide primers for reverse transcription-quantitative polymerase chain reaction analysis.

\begin{tabular}{|c|c|c|}
\hline Gene & $\begin{array}{c}\text { Genbank } \\
\text { accession no. }\end{array}$ & Primer sequence \\
\hline $\mathrm{ZnT}-2$ & RNU50927 & $\begin{array}{l}\text { F, 5'-GGCTGGATCCTGGACTAATGTT-3' } \\
\text { R, 5'-ACACCCCAAAATCCCTTTCTG-3' } \\
\text { Probe, 5'-FAM-CTCACACCACAGCTGGAGAGACACTGAGG-TAMRA-3' }\end{array}$ \\
\hline ZnT-4 & NM_172066.1 & $\begin{array}{l}\text { F, 5'-GCTGAAGCAGAGGAAGGTGAA-3' } \\
\text { R, 5'-TCTCCGATCATGAAAAGCAAGTAG-3' } \\
\text { Probe, 5'-FAM-CAGGCTGACCATCGCTGCCGT-TAMRA-3' }\end{array}$ \\
\hline ZnT-5 & NM_001106404.1 & $\begin{array}{l}\text { F, 5'-CCAGCACATGTCTGGCCTAA-3' } \\
\text { R, 5'-TTTGCAGTACTTCATGGATTCCA-3' } \\
\text { Probe,5'-FAM-CACTGGCTTCCACGATGTCCTGGCTAT-TAMRA-3' }\end{array}$ \\
\hline ZnT-6 & NM_001106708.1 & $\begin{array}{l}\text { F, 5'-CGGCATTATCCCAGGACTCA-3' } \\
\text { R, 5'-CCAGCAAGATCGATCAGAACAA-3' } \\
\text { Probe, 5'-FAM-TTCTTGCCCCGCATGAACCCG-TAMRA-3' }\end{array}$ \\
\hline ZnT-7 & XM_001073594.1 & $\begin{array}{l}\text { F, 5'-TTGGGATCCGCGTCTGA-3' } \\
\text { R, 5'-CCCTCTAGAAGTGACTCGGTATGG-3' } \\
\text { Probe, 5'-FAM-TCGTCTCTGCTGTCACTGCCGCC-TAMRA-3' }\end{array}$ \\
\hline DCT-1 & NM_001108855.1 & $\begin{array}{l}\text { F, 5'-TGGCTGCCTCAGCATCTG-3' } \\
\text { R, 5'-CCCAGTTGTTGAGTGGTTTGG-3' } \\
\text { Probe, 5'-FAM-AAAACCAGAGCCCCTTCCCTACGCA-TAMRA-3' }\end{array}$ \\
\hline ZIP-6 & NM_001024745.1 & $\begin{array}{l}\text { F, 5'-CTCACTGCTGGCTTGTTCATG-3' } \\
\text { R, 5'-CGTGATCACTGGCATCATTGT-3' } \\
\text { Probe, 5'-FAM-TCGCTCTGGTCGACATGGTACCCG-TAMRA-3' }\end{array}$ \\
\hline ZIP-7 & NM_001008885.1 & $\begin{array}{l}\text { F, 5'-GACTTGGCACACAACTTCACAGA-3' } \\
\text { R, 5'-GGACTGTCATCGTGGTCAGGAT-3' } \\
\text { Probe, 5'-FAM-CTGGCCATTGGTGCTTCCTTCCG-TAMRA-3' }\end{array}$ \\
\hline Cathepsin D & NM_134334 & $\begin{array}{l}\text { F, 5'-CATCGCAGCCAAGTTTGATG-3' } \\
\text { R, 5'-CCGGGAGCACATTGTTAACA-3' } \\
\text { Probe, 5'-FAM-CATCTTGGGCATGGGCTACCCTTTTATC-TAMRA-3' }\end{array}$ \\
\hline Cathepsin L & NM_013156 & $\begin{array}{l}\text { F, 5'-GGGTATGGATGGCTACATCAAAA-3' } \\
\text { R, 5'-GGCTGCGGTGGCAAGTC-3' } \\
\text { Probe, 5'-FAM-AGCCAAAGACCGGAACAACCACTGC-TAMRA-3' }\end{array}$ \\
\hline
\end{tabular}

ZnT, zinc transporter; DCT-1, divalent cation transporter 1; ZIP, Zrt-Irt-like protein.

scores were compared using a non-parametric Kruskal-Wallis test. SAS 8.0 statistical software (SAS Institute, Cary, NC, USA) was used to perform statistical analysis. Data is presented as the mean \pm standard deviation. $\mathrm{P}<0.05$ was considered to indicate a statistically significant difference.

\section{Results}

Western blot analysis. Significant increases in the hippocampal protein expression levels of beclin-1 (Fig. 1A) and reductions in those of Bcl-2 (Fig. 1B) were observed at 3, 12 and $24 \mathrm{~h}$ after the last seizures in the RS group. beclin-1 upregulation and Bcl-2 downregulation were mitigated by pretreatment with E-64d.

Timm staining. As shown in Fig. 2A, there were no evident mossy fiber terminals in the control hippocampus. There was prominent aggregation of mossy fiber terminals in the stratum pyramidale of CA3 subfield (Fig. 2B-b) and the dentate gyrus (Fig. 2B-c) compared with the CONT group. In the E64D group rats (Fig. 2C), the aggregation of mossy fiber terminals was markedly decreased in the supragranular region of dentate gyrus and CA3 subfield (Fig. 2C-b and 2C-c) compared with the RS group.

$R T-q P C R$ analysis. RT-qPCR was employed to evaluate the relative mRNA expression levels of ZnT-2,ZnT-4,ZnT-5,ZnT-6, ZnT-7, DCT-1, ZIP-6, ZIP-7, cathepsin D and cathepsin L in the hippocampus at P51. As shown in Fig. 3A, RS group rats showed a significant upregulation of the mRNA expression of ZnT-4,ZnT-5, ZIP-7 and cathepsin D compared with the CONT group rats $(\mathrm{P}<0.05)$. In addition, there was long-term downregulated expression of ZnT-4, ZIP-6, ZIP-7 and cathepsin L in the E64D group rats compared with the RS group $(\mathrm{P}<0.05)$.

Linear correlation analysis showed a total of 45 Pearson correlation coefficients among the ten genes. As shown in Fig. 3B and Table II, 14 of the Pearson correlation coefficients showed significant inter-relationship $(\mathrm{P}<0.05)$; primarily among ZIP-7, ZnT-4, ZnT-5, ZnT-7, cathepsin D and cathepsin L. The results suggest that the ZnTs/ZIP7/cathepsin signaling pathway may serve a key role in the neuroprotective effects of E-64d. 
A

Expression of Beclin1 in hippocampus
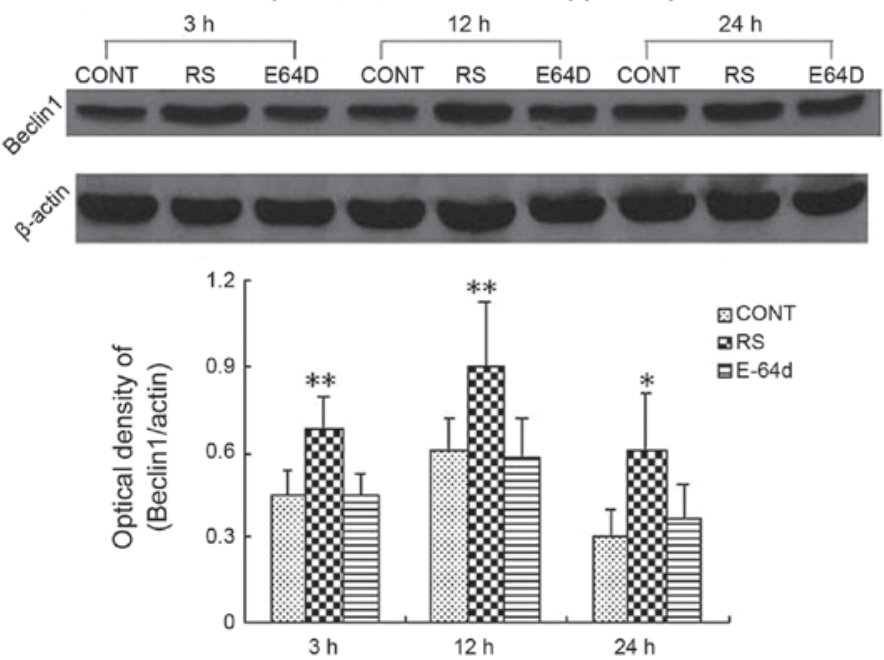

B Expression of Bcl-2 in hippocampus
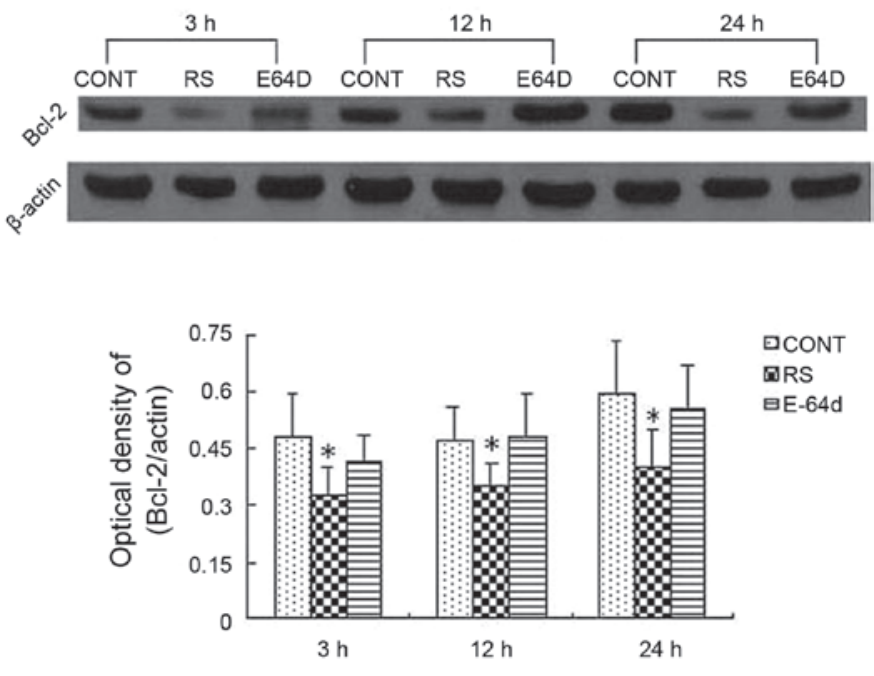

Figure 1. Western blot analysis of (A) beclin-1 and (B) Bcl-2 protein expression levels in the rat hippocampus at 3, 12 and $24 \mathrm{~h}$ after the last seizures. Data are expressed as the mean \pm standard deviation. Data were analyzed with post-hoc comparisons using a Bonferroni test after analysis of variance." $\mathrm{P}<0.05$ and ${ }^{* *} \mathrm{P}<0.01$, vs. CONT and E64D groups. CONT, control group; RS, recurrent seizure group; E64D, E-64d-treated group; Bcl-2, B-cell lymphoma 2.

A a
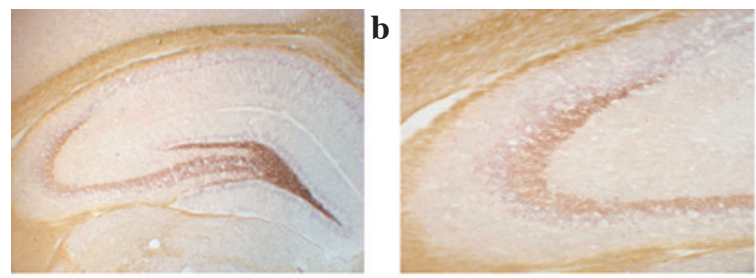

B a

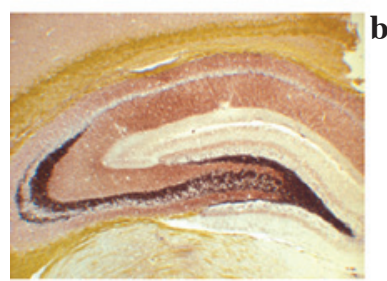

C a
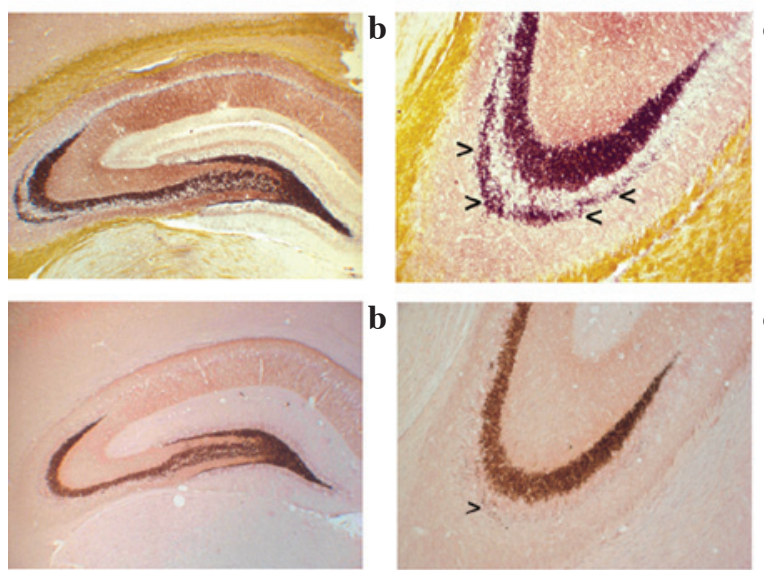

D

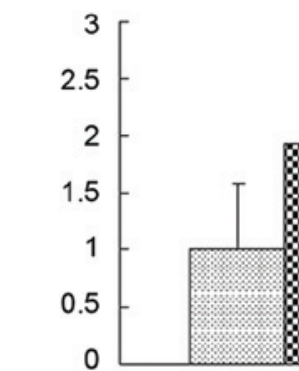

Timm score
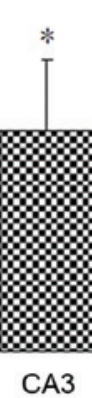

CA3
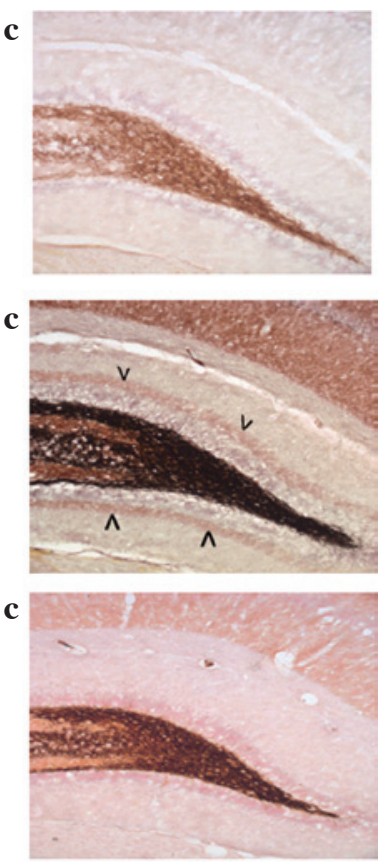

MCONT RSEE-64D

Figure 2. Representative images of mossy fiber sprouting by Timm staining in the (A) CONT, (B) RS and (C) E64D groups. Parts labeled as (a) represent panoramic view of hippocampus from CONT to E4D; (b) represent CA3 subfield from CONT to E64D; similarly, parts labeled as (c) represent dentate gyrus subfield. Excessive quantities of Timm staining are observed in the stratum pyramidale of CA3 subfield (as shown in B-b) and in the inner molecular layer of the granule cells (as shown in B-b) in the RS group (arrows). Mild quantities of Timm staining are observed in the stratum pyramidale of CA3 subfield (as shown in C-b) in the E64D group (arrow). Data are expressed as the mean \pm standard deviation. (D) Timm staining scores compared using a non-parametric Kruskal-Wallis test. Magnification: (a) x40; (b) and (c) x100. "P<0.05 vs. CONT group; ${ }^{*} \mathrm{P}<0.05$ vs. RS group. CONT, control group; RS, recurrent seizure group; E64D, E-64d-treated group. 
A

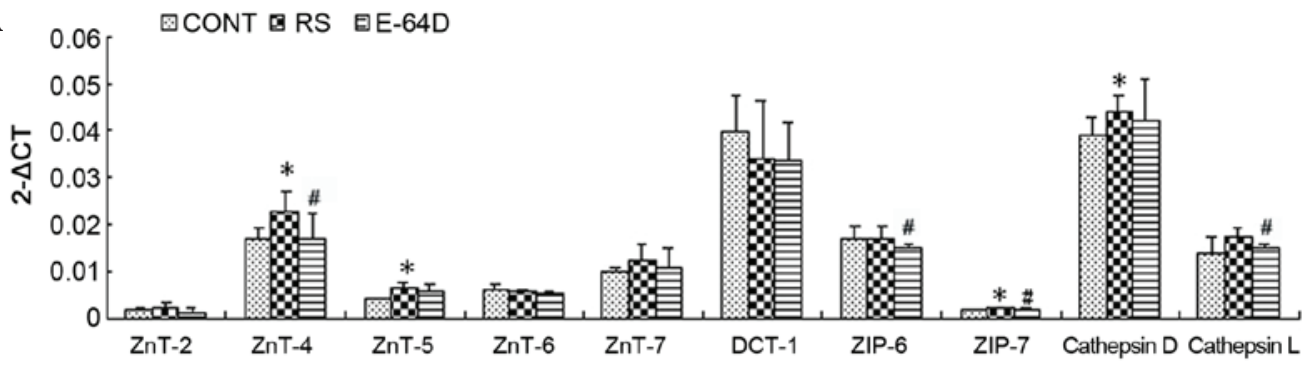

B

Realtime quantitive PCR with 2- $\triangle \mathrm{CT}$ method

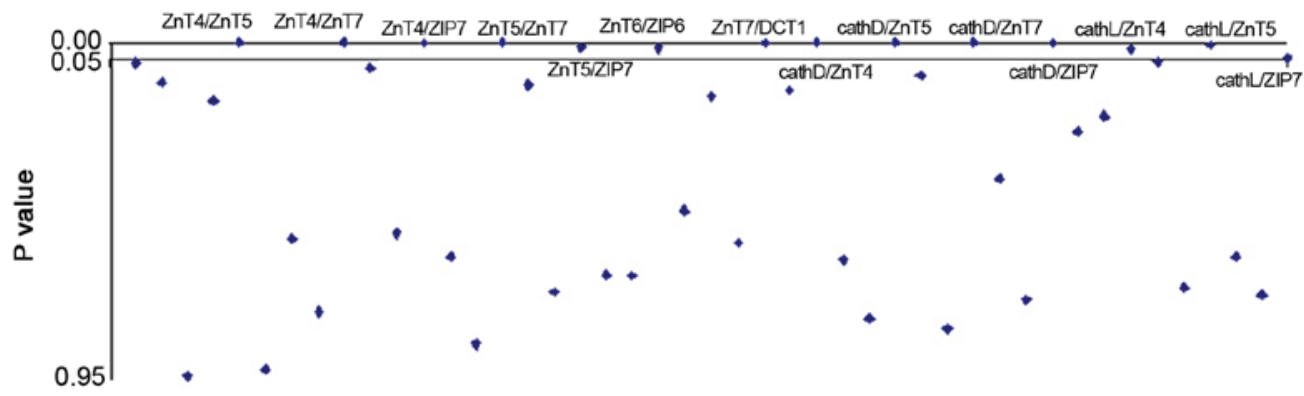

Figure 3. mRNA expression levels as identified using reverse transcription-quantitative polymerase chain reaction analysis. (A) Expression of ZnT-2, ZnT-4, ZnT-5, ZnT-6, ZnT-7, DCT-1, ZIP-6, ZIP-7, cathepsin D and cathepsin L in the rat hippocampus after behavioral analysis. (B) Pearson's linear correlation analyses of the ten genes. Data are expressed as the mean \pm standard deviation. Data was analyzed with post hoc comparisons using a Bonferroni test after analysis of variance. ${ }^{*} \mathrm{P}<0.05$ vs. CONT group; ${ }^{\#} \mathrm{P}<0.05$ vs. RS group. CONT, control group; RS, recurrent seizure group; E64D, E-64d-treated group; Znt, zinc transporter; DCT-1, divalent cation transporter 1; ZIP, Zrt-Irt-like protein.

Table II. Linear correlation analysis among the 10 genes, showing the 14 Pearson correlation coefficients $(r)$ with statistically significant inter-associations.

\begin{tabular}{lcl}
\hline Associated genes & Coefficient value & P-value \\
\hline ZnT-4/ZnT-5 & 0.90 & $<0.0001$ \\
ZnT-4/ZnT-7 & 0.89 & $<0.0001$ \\
ZnT-4/ZIP-7 & 0.64 & $<0.01$ \\
ZnT-5/ ZnT-7 & 0.90 & $<0.0001$ \\
ZnT-5/ZIP-7 & 0.57 & $<0.05$ \\
ZnT-6/ZIP-6 & 0.56 & $<0.05$ \\
ZnT-7/DCT-1 & 0.64 & $<0.01$ \\
Cathepsin D/ZnT-4 & 0.72 & $<0.001$ \\
Cathepsin D/ZnT-5 & 0.74 & $<0.001$ \\
Cathepsin D/ZnT-7 & 0.70 & $<0.01$ \\
Cathepsin D/ZIP-7 & 0.62 & $<0.01$ \\
Cathepsin L/ZnT-4 & 0.53 & $<0.05$ \\
Cathepsin L/ZnT-5 & 0.60 & $<0.01$ \\
Cathepsin L/ZIP-7 & 0.47 & $<0.05$ \\
\hline
\end{tabular}

ZnT, zinc transporter; ZIP, Zrt-Irt-like protein 6; DCT, divalent cation transporter.

\section{Discussion}

E-64d is an autophagy inhibitor that has been shown to perform a neuroprotective function in spinal cord injury, hypoxic-ischemic brain injury, acute optic neuritis (14-17), in addition to kainic acid-induced excitotoxicity of primary striatal neurons (18). However, the molecular mechanisms underlying the efficacy of E-64d remain unclear. The aim of the present study was to elucidate the neuroprotective effects of autophagy inhibitors on developmental seizure-induced brain damage by neuropathological and molecular biology analyses, focusing on ZnT-associated gene expression in the hippocampus.

In accordance with previous results (19), pretreatment with E-64d prior to the induction of seizures markedly attenuated the long-term hippocampal mossy fiber sprouting. Furthermore, E-64d treatment resulted in the downregulated mRNA expression of ZnT-4, ZIP-6, ZIP-7 and cathepsin L at the long-term time point compared with the RS group. To the best of our knowledge, the present study is the first to investigate the long-term effects of an autophagy inhibitor on the expression profiles of ZnTs in hippocampus following developmental seizures.

Previous results showed that CBI, an autophagy inhibitor, could improve functional deficits and inhibit hippocampal sprouting by modulating long-term PRG-1 expression in the hippocampus and cerebral cortex (9). In another study (10), we reported for the first time that pretreatment with 3-MA, another autophagy inhibitor, markedly attenuated neonatal seizure-induced ZnT-1, ZnT-2, LC3 and beclin-1 elevations in the hippocampus at the acute phase time point following seizures. However, the present study primarily examined the ZnT-associated mRNA expression at a long-term time point following seizures during adolescence (P51), a time point which is in parallel with the genesis of mossy fiber sprouting in hippocampus. The results suggest that the regulators for zinc homeostasis may be involved in E-64d-mediated neuroprotection following developmental seizure-induced excitotoxicity. 
The divalent cation $\mathrm{Zn}^{2+}$, an essential trace element that performs structural and cofactor functions, is concentrated in synaptic vesicles, particularly in the hippocampal zinc-rich mossy fiber pathway, which has the highest quantity of zinc in brain (20). The presence of this type of vesicular zinc in the mossy fiber system may be detected using Timm staining, where its excessive synaptic movement from pre- to post-synaptic neurons via ZnTs contributes to regenerative sprouting following epilepsy (21). Among the ten ZnTs that have been identified to date, ZnT-2, ZnT-4, ZnT-5, ZnT-6 and ZnT-7 are of particular interest due to their function in intracellular organelles. ZnT-2 and ZnT-4 transport zinc into endosomes and lysosomes, while ZnT-5, ZnT-6 and ZnT-7 appear to be localized on the Golgi apparatus (22). A study by McCormick and Kelleher indicated that ZnT-4 provides zinc to zinc-dependent proteins in the trans-Golgi network, which directly contributed to labile zinc accumulation as ZnT-4 overexpression increased FluZin3 (zinc probe used to monitor zinc fluxes during fluorescent imaging) fluorescence (23). As FluZin3 is regarded as a marker for detecting sprouting mossy fibers in the temporal lobe epileptic hippocampus (24), it is possible that ZnT-4 overexpression in the brain may be a molecular marker for hippocampal mossy fiber sprouting. The present results showed the upregulation of the transcription of ZnT-4 in the RS group compared with the CONT group. Notably, this elevated mRNA expression of ZnT-4 was significantly downregulated by pretreatment with E-64d. As mentioned above, ZnT-4 transports zinc into endosomes and lysosomes, which are also the target organelles for autophagy inhibitors. The present results indicate that ZnT-4 may contribute to E-64d-mediated improvement of morphological and cognitive functions, which merits further investigation. In addition, this study identified significantly increased mRNA expression of ZnT-5 and a increasing trend of ZnT-7 in the RS group compared with the CONT group. Furthermore, linear correlation analysis showed significant inter-relationships among ZnT-4, ZnT-5 and ZnT-7. Combined with previous results, which suggest that ZnT-5 and ZnT-7 contribute synergic to the activation of phosphatases in cytoplasmic membrane (25), it is reasonable to speculate that ZnT-4, ZnT-5 and ZnT-7 may contribute synergically to the neuroprotective effects of E-64d in an animal model of penicillin-induced developmental seizures.

Apart from ZnTs, the regulation of cellular zinc homeostasis is controlled closely by a number of different mechanisms. Hence, we further investigated the expression of ZIP-6, ZIP-7 and DCT-1 in hippocampus. ZIP is responsible for the movement of zinc into a cell from the extracellular space, and transports zinc into the cytoplasm from organelles that contain zinc (22). Among the 14 mammalian members of the ZIP family, the ZIP transporters ZIP-6 and ZIP-7 have been detected in the brain (26). DCT-1 (also known as divalent metal transporter 1) is present in the hippocampal pyramidal and granule cells, cerebellar granule cells and pyramidal cells of the piriform cortex in high densities (27). In the present study, ZIP-7 showed significantly upregulated mRNA expression in the RS group compared with CONT group rats, while no significant differences were observed in the mRNA levels of ZIP-6 and DCT-1 between the RS and CONT groups. In addition, the upregulated expression of ZIP-7 was significantly inhibited by pretreatment with E-64d. Furthermore, linear correlation analysis demonstrated significant inter-relationships between ZIP-7 and ZnT-4 or ZnT-5. This result suggests that ZIP-7 is correlated with the long-term hippocampal mossy fiber sprouting and cognitive processing following developmental seizures. Previous studies have reported that ZIP-7 plays a critical role in zinc homeostasis (28), and is implicated in aberrant cellular biochemical signaling such as tyrosine kinase activation and growth factor signaling $(29,30)$. Collectively, these results suggest that ZIP-7 functions as a critical node in zinc-mediated aberrant mossy fiber sprouting in hippocampus and may be a novel target for the intervention effect of E-64d following developmental seizure-induced brain damage.

A previous study by Luo et al (31) showed that the inhibition of autophagy by 3-MA and bafliomycin A1 could reduce traumatic brain injury (TBI)-induced cell damage and attenuate behavioral outcomes via the inhibition of the TBI-induced upregulation of LC3, beclin-1 and the beclin-1/Bcl-2 ratio in the hippocampus. The present results also observed the modulatory effects of E-64d on beclin-1 and Bcl-2, which were observed at 3 and $12 \mathrm{~h}$ after the last seizures. Notably, the present RT-qPCR results showed upregulated cathepsin D expression in the RS group compared with the CONT group. Furthermore, pretreatment with E-64d significantly inhibited the elevated mRNA expression of cathepsin L in comparison with the RS group rats. Cathepsin D and cathepsin L are acid lysosomal hydrolases. It has been reported that the majority of zinc-containing vacuoles of cultured retinal cells are lysosomes, and that the accumulation of zinc in lysosomes induced the release of cathepsin D into the cytosol, which may be a key mechanism of ethambutol-induced retinal cell death (32). These results are in accordance withe present study, in which the expression of ZnT-4, ZnT-5, ZIP-7 and cathepsin D was upregulated in RS group rats compared with CONT group rats. In addition, long-term downregulated expression of ZnT-4, ZIP-6, ZIP-7 and cathepsin L was detected in E64D group rats compared with the RS group. Linear correlation analysis further indicated significant inter-relationships among ZIP-7, ZnT-4, ZnT-5, ZnT-7, cathepsin D and cathepsin L. Based on these findings, it is postulated that perturbations of intralysosomal zinc and cathepsin metabolism, and the subsequent results in lysosomal dysfunction, may underlie the neuronal dysfunction characteristic of developmental seizure-induced brain damage. Thus, the lysosomal modulation by E-64d represents a novel approach for treating brain damage induced by developmental seizures.

In conclusion, the present study provides insights into the abnormalities in signal transduction of zinc, reflected by ZnTs and ZIP. These results suggest that the E-64d attenuates hippocampal mossy fiber sprouting, at least in part, via the modulation of the ZnT-4/ZIP-7/cathepsin signaling pathway. These results may offer a novel strategy for the development of therapeutic interventions for treatment of developmental seizure-induced brain damage.

\section{Acknowledgements}

This study was supported by the National Natural Science Foundation of China (grant nos. 81271458 and 81471337) and the Jiangsu Province's Key Provincial Talents Program 
(grant no. RC2011113), a project funded by the Priority Academic Program Development of Jiangsu Higher Education Institutions.

\section{References}

1. Au AK, Bayir H, Kochanek PM and Clark RS: Evaluation of autophagy using mouse models of brain injury. Biochim Biophys Acta 1802: 918-923, 2010.

2. Bae N, Ahn T, Chung S, Oh MS, Ko H, Oh H, Park G and Yang HO: The neuroprotective effect of modified Yeoldahanso-tang via autophagy enhancement in models of Parkinson's disease. J Ethnopharmacol 134: 313-322, 2011

3. Jing CH, Wang L, Liu PP, Wu C, Ruan D and Chen G: Autophagy activation is associated with neuroprotection against apoptosis via a mitochondrial pathway in a rat model of subarachnoid hemorrhage. Neuroscience 213: 144-153, 2012.

4. Holopainen IE: Seizures in the developing brain: Cellular and molecular mechanisms of neuronal damage, neurogenesis and cellular reorganization. Neurochem Int 52: 935-947, 2008.

5. Dulac O, Nabbout R, Plouin P, Chiron C and Scheffer IE: Early seizures: Causal events or predisposition to adult epilepsy? Lancet Neurol 6: 643-651,2007.

6. Holmes GL: Effects of seizures on brain development: Lessons from the laboratory. Pediatr Neurol 33: 1-11, 2005.

7. Ni H, Jiang YW, Bo T, Wang JM and Wu XR: C-Fos, N-methyl-D-aspartate receptor 2C, GABA-A-alpha 1 immonoreactivity, seizure latency and neuronal injury following single or recurrent neonatal seizures in hippocampus of Wistar rat. Neurosci Lett 380: 149-154, 2005.

8. Ni H, Jiang YW, Tao LY, Cen JN and Wu XR: Effects of penicillin-induced developmental epilepticus on hippocampal regenerative sprouting, related gene expression and cognitive deficits in rats. Toxicol Lett 188: 161-166, 2009.

9. Ni H, Yan JZ, Zhang LL, Feng X and Wu XR: Long-term effects of recurrent neonatal seizures on neurobehavioral function and related gene expression and its intervention by inhibitor of cathepsin B. Neurochem Res 37: 31-39, 2012.

10. Ni H, Feng X, Gong Y, Tao LY and Wu XR: Acute phase expression pattern of ZnTs, LC3 and beclin-1 in rat Hippocampus and its regulation by 3 -methyladenine following recurrent neonatal seizures. Biol Trace Elem Res 143: 320-331, 2011.

11. Luo CL, Chen XP, Yang R, Sun YX, Li QQ, Bao HJ, Cao QQ, $\mathrm{Ni}$ H, Qin ZH and Tao LY: Cathepsin B contributes to traumatic brain injury-induced cell death through a mitochondria-mediated apoptotic pathway. J Neurosci Res 88: 2847-2858, 2010.

12. Ni H, Jiang YW, Tao LY, Jin MF and Wu XR: ZnT-1, ZnT-3, CaMKII, PRG-1 expressions in hippocampus following neonatal seizure-induced cognitive deficit in rats. Toxicol Lett 184 $145-150,2009$

13. Johnson MR, Wang K, Smith JB, Heslin MJ and Diasio RB: Quantitation of dihydropyrimidine dehydrogenase expression by real-time reverse transcription polymerase chain reaction. Anal Biochem 278: 175-184, 2000.

14. Ray SK, Matzelle DC, Wilford GG, Hogan EL and Banik NL: E-64-d prevents both calpain upregulation and apoptosis in the lesion and penumbra following spinal cord injury in rats. Brain Res 867: 80-89, 2000.

15. Ray SK, Matzelle DD, Wilford GG, Hogan EL and Banik NL: Cell death in spinal cord injury (SCI) requires de novo protein synthesis. Calpain inhibitor E-64-d provides neuroprotection in SCI lesion and penumbra. Ann NY Acad Sci 939: 436-449, 2001.
16. Tsubokawa T, Solaroglu I, Yatsushige H, Cahill J, Yata K and Zhang JH: Cathepsin and calpain inhibitor E64d attenuates matrix metalloproteinase-9 activity after focal cerebral ischemia in rats. Stroke 37: 1888-1894, 2006.

17. Tsubokawa T, Yamaguchi-Okada M, Calvert JW, Solaroglu I, Shimamura N, Yata K and Zhang JH: Neurovascular and neuronal protection by E64d after focal cerebral ischemia in rats. J Neurosci Res 84: 832-840, 2006.

18. Dong XX, Wang YR, Qin S, Liang ZQ, Liu BH, Qin ZH and Wang Y: P53 mediates autophagy activation and mitochondria dysfunction in kainic acid-induced excitotoxicity in primary striatal neurons. Neuroscience 207: 52-64, 2012.

19. Ni H, Ren SY, Zhang LL, Sun Q, Tian T and Feng X: Expression profiles of hippocampal regenerative sprouting-related genes and their regulation by E-64d in a developmental rat model of penicillin-induced recurrent epilepticus. Toxicol Lett 217: 162-169, 2013.

20. Takeda A: Zinc signaling in the hippocampus and its relation to pathogenesis of depression. Mol Neurobiol 44: 166-174, 2011.

21. Mitsuya K, Nitta N and Suzuki F: Persistent zinc depletion in the mossy fiber terminals in the intrahippocampal kainate mouse model of mesial temporal lobe epilepsy. Epilepsia 50: 1979-1990, 2009.

22. Nakashima AS and Dyck RH: Zinc and cortical plasticity. Brain Res Rev 59: 347-373, 2009.

23. McCormick NH and Kelleher SL: ZnT4 provides zinc to zinc-dependent proteins in the trans-Golgi network critical for cell function and $\mathrm{Zn}$ export in mammary epithelial cells. Am J Physiol Cell Physiol 303: C291-C297, 2012.

24. Sutula TP and Dudek FE: Unmasking recurrent excitation generated by mossy fiber sprouting in the epileptic dentate gyrus: An emergent property of a complex system. Prog Brain Res 163: 541-563, 2007.

25. Suzuki T, Ishihara K, Migaki H, Matsuura W, Kohda A, Okumura K, Nagao M, Yamaguchi-Iwai Y and Kambe T: Zinc transporters, ZnT5 and ZnT7, are required for the activation of alkaline phosphatases, zinc-requiring enzymes that are glycosylphosphatidylinositol-anchored to the cytoplasmic membrane. J Biol Chem 280: 637-643, 2005.

26. Lichten LA and Cousins RJ: Mammalian zinc transporters: Nutritional and physiologic regulation. Annu Rev Nutr 29: 153-176, 2009.

27. Mims MP and Prchal JT: Divalent metal transporter 1. Hematology 10: 339-345, 2005.

28. Yan G, Zhang Y, Yu J, Yu Y, Zhang F, Zhang Z, Wu A, Yan X, Zhou Y and Wang F: Slc39a7/zip7 plays a critical role in development and zinc homeostasis in zebrafish. PLoS One 7: e42939, 2012.

29. Taylor KM, Vichova P, Jordan N, Hiscox S, Hendley R and Nicholson RI: ZIP7-mediated intracellular zinc transport contributes to aberrant growth factor signaling in antihormone-resistant breast cancer Cells. Endocrinology 149: 4912-4920, 2008

30. Hogstrand C, Kille P, Nicholson RI and Taylor KM: Zinc transporters and cancer: A potential role for ZIP7 as a hub for tyrosine kinase activation. Trends Mol Med 15: 101-111, 2009.

31. Luo CL, Li BX, Li QQ, Chen XP, Sun YX, Bao HJ, Dai DK, Shen YW, Xu HF, Ni H, et al: Autophagy is involved in traumatic brain injury-induced cell death and contributes to functional outcome deficits in mice. Neuroscience 184: 54-63, 2011.

32. Chung H, Yoon YH, Hwang JJ, Cho KS, Koh JY and Kim JG: Ethambutol-induced toxicity is mediated by zinc and lysosomal membrane permeabilization in cultured retinal cells. Toxicol Appl Pharmacol 235: 163-170, 2009. 